Acta Physica Academiae Scientiarum Hungaricae, Tomus 42 (3), pp. $185-187$ (1977)

\title{
ADDENDUM TO THE "CORRECTION OF ELECTRON CAPTURE RATIOS MEASURED BY MULTI-WIRE PROPORTIONAL COUNTER" [1]
}

\author{
By \\ E. VAtaI \\ INSTITUTE OF NUCLEAR RESEARCH OF THE HUNGARIAN ACADEMY OF SCIENCES, DEBRECEN
}

(Received 28. I. 1977)

The formulas for the calculation of the corrections of multi-wire proportional counters published in [1] are explained, simplified and corrected.

The corrections of the multi-wire proportional counter calculated by us or by using the formulas given in [1] were used in numerous cases in the last 7 years (e.g. [2], [3]). Their use made it possible to increase accuracy and reliability, which was especially important in the measurements of the $M / L$ capture ratios. These corrections are superior to the similar calculations [5] based on the formulas of reactor theory not only because they are calculated for the actual shape of the counter, but because the integrations are performed for all necessary variables without simplification. Such calculations are needed nowadays also [4], therefore the programs have been re-written and the calculations repeated. The results agree with those of previous calculations, nevertheless it became clear that 1) a better explanation of the formulas of [1] is necessary to promote application; 2) in some cases the functions of integration can be simplified; 3) some clerical errors in the formulas should be corrected. These problems are solved below.

Two sections of the counter are given in Fig. 1 to clear the system of coordinates and notations used. The counter is given in cylindrical coordinates $\left(z, r, \varphi^{\prime}\right)$. The direction of the radiation (X-ray) from a given point of the counter is described by spherical angles $\varphi$ changing from $-\pi$ to $+\pi$ in the plane perpendicular to the axis of the counter $z$, and $\Theta$ changing from 0 to $\pi$ in the plane determined by $\varphi$ and parallel with $z$. The values of $\Theta_{i}$ in Fig. 1 are given for anticoincidence triggering.

The self absorbtion and emission of $\mathrm{X}$-rays from the counter are given by integrals of the following type:

$$
I=\frac{1}{4 \pi V} \int_{z_{1}}^{z_{2}} \mathrm{~d} z \int_{r_{1}}^{r_{2}} r \mathrm{~d} r \int_{0}^{2 \pi} \mathrm{d} \varphi^{\prime} \int_{0}^{\pi} \sin \Theta \mathrm{d} \Theta \int_{-\pi}^{\pi} \mathrm{d} \varphi f(z, r, \Theta, \varphi),
$$

which is valid for homogeneous distribution of activity and when the escape 


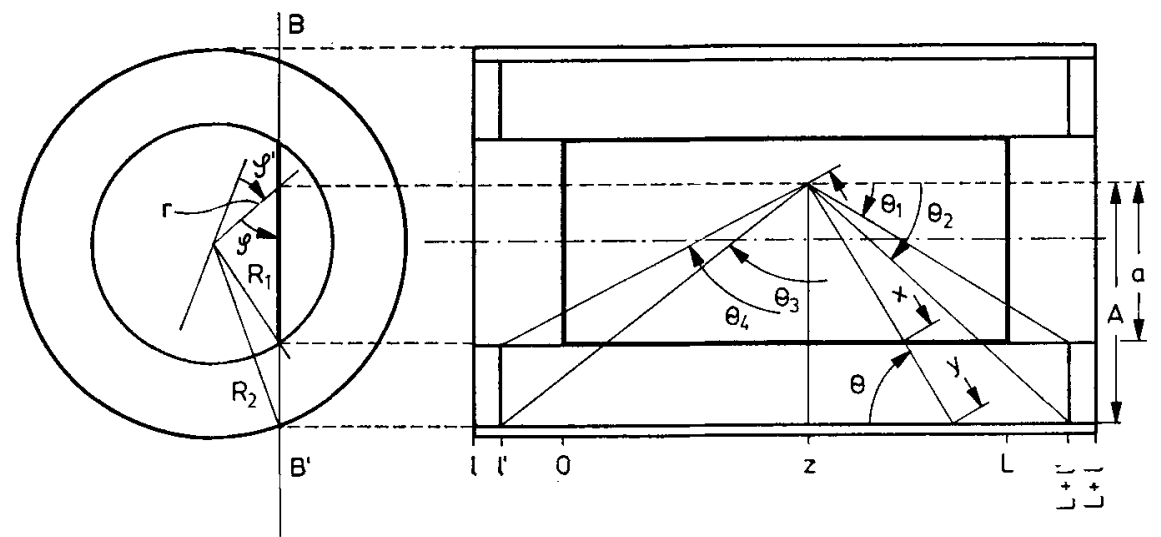

Fig. 1. Multi-wire proportional counter in two sections to explain the notations used

of secondary radiation from the given part of the counter can be neglected. Due to cylindrical symmetry, $f$ is independent of $\varphi^{\prime}$, which makes analytical integration feasible for this coordinate.

Below we give the intervals and functions of integration.

Notations

$$
\begin{aligned}
& a=r \cos \varphi+\left(R_{1}^{2}-r^{2} \sin ^{2} \varphi\right)^{1 / 2} \\
& b=r \cos \varphi-\left(R_{1}^{2}-r^{2} \sin ^{2} \varphi\right)^{1 / 2}
\end{aligned}
$$

and

are used.

$$
A=r \cos \varphi+\left(R_{2}^{2}-r^{2} \sin ^{2} \varphi\right)^{1 / 2}
$$

1. Escape from the central counter is an integral for $0 \leqq z \leqq L ; 0 \leqq r \leqq R_{1}$ and $-\pi \leqq \varphi \leqq \pi$. The function $f=\exp (-\mu x)$ where $x$ has different forms for the three intervals of $\Theta$ :

$$
\begin{array}{ll}
x=(L-z) / \cos \Theta & \text { if } 0 \leqq \operatorname{tg} \Theta \leqq a /(L-z) ; \\
x=a / \sin \Theta & \text { if } a /(L-z) \leqq \operatorname{tg} \Theta \leqq a / z ; \\
x=z /|\cos \Theta| & \text { if } a / z \leqq \operatorname{tg} \Theta \leqq \pi
\end{array}
$$

2. Anticoincidence triggering can be obtained by subtraction from the radiation unabsorbed in the central counter $[\propto \exp (-\mu x)]$ the one unabsorbed both in the central and anticoincidence counters $[\propto \exp (-\mu y)]$ i.e. $f=$ $=\exp (-\mu x)-\exp (-\mu y)$. Functions of the same form are to be used for all cases below. Integration limits for $z, r$ and $\varphi$ are the same as in case 1 . The value $x=a / \sin \Theta$ is the same for all $\Theta-\mathrm{s}$, while

$$
\begin{array}{ll}
y=\left(L+l^{\prime}-z\right) / \cos \Theta & \text { if } a /\left(L+l^{\prime}-z\right) \leqq \operatorname{tg} \Theta \leqq A /\left(L+l^{\prime}-z\right)=D_{2} \\
y=A / \sin \Theta & \text { if } D_{2} \leqq \operatorname{tg} \Theta \text { and } \operatorname{tg}(\Theta-\pi / 2) \leqq\left(l^{\prime}+z\right) / A=D_{3} \\
y=\left(l^{\prime}+z\right) /|\cos \Theta| & \text { if } D_{3} \leqq \operatorname{tg}(\Theta-\pi / 2) \leqq\left(l^{\prime}+z\right) / a
\end{array}
$$


3. Replacement is the radiation originated outside the central counter but absorbed in it. We divide the corresponding part of the counter into three parts according to the forms of $x$ and $y$.

3a. Replacement from the part of anticoincidence counters, neighbouring with the central counter is an integral for $\sin |\varphi| \leqq r / R_{1}$, because radiation emitted under larger $\varphi$ does not enter into the central counter. $R_{1} \leqq r \leqq R_{3}, R_{3}$ being the radius of the container and $0 \leqq z \leqq L ; x=$ $=b / \sin \Theta$ for all values of $\Theta$, while

$$
\begin{array}{ll}
y=(L-z) / \cos \Theta & \text { if } b /(L-z) \leqq \operatorname{tg} \Theta \leqq a /(L-z)=D_{2} \\
y=a / \sin \Theta & \text { if } D_{2} \leqq \operatorname{tg} \Theta \text { and } D_{3}=a / z \gtreqless \operatorname{tg}(\Theta-\pi / 2) \\
y=z /|\cos \Theta| & \text { if } D_{3} \leqq \operatorname{tg}(\Theta-\pi / 2) \leqq b / z
\end{array}
$$

3b. Replacement from the ends of the anticoincidence counters is an integral for the same intervals of $\varphi$ and $r$ as in $3 a$, and $-l \leqq z \leqq 0$. The integral for $L \leqq z \leqq L+l$ is the same. Beside dividing the integral for $\Theta$ into three parts, it is necessary to distinguish between the cases when $a /(L-z) \lessgtr$ $\leq \boldsymbol{b} /|\boldsymbol{z}|$.

$$
\begin{array}{ll}
x=b / \sin \Theta ; y=(L-z) / \cos \Theta & \text { if } b /(L-z) \leqq \operatorname{tg} \Theta \leqq a /(L-z) \text { and } b /|z| \\
x=b / \sin \Theta ; y=a / \sin \Theta & \text { if } a /(L-z) \leqq \operatorname{tg} \Theta \leqq b /|z| .
\end{array}
$$

When this condition cannot be fulfilled because $a /(L-z)>b /|z|$, then $x=$ $=|z| / \cos \Theta ; y=(L-z) / \cos \Theta$ for $b /|z| \leqq \operatorname{tg} \Theta \leqq a /(L-z)$. The functions are the same for both cases in the last interval:

$$
x=|z| / \cos \Theta ; y=a / \sin \Theta \text { if } b /|z| \text { and } a /(L-z) \leqq \operatorname{tg} \Theta \leqq a /|z|
$$

3c. Replacement from the end of the central counter is integral for the same intervals as in case 1 for $r$ and $\varphi$, and as in case $3 b$ for $z$. The integration for $\Theta$ is separated into two parts:

$$
\begin{array}{ll}
x=|z| / \cos \Theta ; y=(L-z) / \cos \Theta & \text { if } 0 \leqq \operatorname{tg} \Theta \leqq a /(L-z) \\
x=|z| / \cos \Theta ; y=a / \sin \Theta & \text { if } a /(L-z) \leqq \operatorname{tg} \Theta \leq a /|z|
\end{array}
$$

The author is grateful to Prof. D. BerÉNyi, Director of the Institute, for his interest in this work.

\section{REFERENCES}

1. E. VAtai, Acta Phys. Hung., 28, 103, 1970.

2. H. Genz, J. P. Renier, J. G. Pengra and R. W. Fink, Phys. Rev., C3, 172, 1971.

3. J. G. Pengra, Second Internat. Conf. on Inner Shell Ionization Phenomena. Abstract of contributed papers, Freiburg, March 29-April 2, 1976. p. 194.

4. K.-W. HofrmanN, private communication, 1976.

5. R. B. Moler and R. W. Finx, Phys. Rev., 131, 821, 1963. 